\title{
Accurate Performance Bounds for Target Detection in WSNs with Deterministic Node Placement
}

\author{
Paolo Medagliani, ${ }^{\# 1}$ Jérémie Leguay, ${ }^{* 2}$ Gianluigi Ferrari, ${ }^{\# 3}$ Vincent Gay, ${ }^{* 4}$ Mario Lopez-Ramos ${ }^{* 5}$ \\ \# Wireless Ad-hoc and Sensor Networks (WASN) Laboratory, University of Parma \\ viale G.P. Usberti 181/A, Parma, Italy \\ $\left\{{ }^{1}\right.$ paolo.medagliani, ${ }^{3}$ gianluigi.ferrari\}@unipr.it \\ * Thales Communications \\ 160 Bd de Valmy, Colombes Cedex, France \\ \{2jeremie.leguay, ${ }^{4}$ vincent.gay, ${ }^{5}$ mario.lopezramos $\}$ @fr.thalesgroup.com
}

\begin{abstract}
This paper addresses target detection applications for long-lasting surveillance of areas of interest using unattended Wireless Sensor Networks (WSNs). In this context, to achieve a long system lifetime, sensing and communication modules of wireless sensor nodes may be switched on and off according to a prefixed duty cycle, whose use has an impact on (i) the latency of notifications (depending only on the communication duty cycle) and (ii) the probability of target detection (depending only on the number of deployed nodes and the sensing duty cycle). This paper provides an accurate evaluation of the probability of missed target detection in scenarios where node positions are known and duty cycles are used to save energy. This work extends a previous work with stochastic node placement [1], and allows to derive simple, yet very accurate, upper and lower bounds on the probability of target detection.
\end{abstract}

\section{INTRODUCTION}

Wireless Sensor Networks (WSNs) are commonly used for environmental monitoring, military surveillance, and industrial automation. These devices are typically composed of embedded microcontrollers with limited data storage capabilities, radio transceivers, physical transducers that sense the environment, and run, most of the time, on batteries. Recent advances in hardware miniaturization, low-power radio communications, and battery lifetime, together with the increasing affordability of such devices, are paving the road for a widespread use of WSNs in a wide range of applications.

When integrated with heterogeneous surveillance systems, in complement to traditional high-power and bulky observation sub-systems (e.g., mounted optronic systems and radars), hundreds of tiny sensor nodes can help secure and protect people and assets in remote or inaccessible areas. Through the use of embedded transducers, such as acoustic, seismic or infrared sensors, they can perform local or collaborative target signature detection and classification, as well as trigger actuators (e.g., flash lights, sirens). These nodes can be easily deployed and recovered, are lightweight, and provide costeffective complements to existing surveillance systems. A WSN can be exposed as a sub-system to the rest of the information system through gateway nodes, which can offer backhaul connectivity and have more capabilities in terms of storage and processing. Most of the sensors are inherently resource-constrained because of their size and cost, and this, in turns, imposes limits in terms of energy, computational speed, storage capacity, and communication bandwidth.

In the literature, a few papers address in detail the problem of target detection and decision reporting. In [2], the authors present the design and the implementation of a monitoring system, referred to as VigilNet, based on a WSN. The authors derive an energy-efficient adaptive surveillance strategy and validate it through experimental tests. In [3], under the assumptions that the road map is known and the target movement is confined into roads, the authors describe an algorithm, referred to as Virtual Scanning Algorithm, which guarantees that the incoming target will be detected before reaching a given protection point. In [4], the authors provide some insights about random/deterministic node placement strategies to guarantee a minimum network coverage. However, these approaches do not provide a performance description in terms of probability of missed target detection, when sensing duty cycles at the nodes are considered.

This paper addresses the problem of target detection using a long-term deployment of an unattended WSN over large monitored area. In this context, one of the main design goals is to maximize the operational lifetime of the systemtypically by periodically switching on and off sensing and communication interfaces-while ensuring that intruders will eventually be detected. In particular, we focus on the derivation of an analytical framework for efficiently selecting the length of these duty-cycles, in order to operate at a desired operational point, characterized by a trade-off between energy consumption and quality of service (in terms of detection capability). In [1], a stochastic node deployment has been considered and an average performance analysis has been carried out. In this paper, instead, we consider a deterministic node placement (i.e., their positions are known) and derive accurate lower and upper bounds for the probability of target detection. These results allow to estimate more precisely the performance of a given deployment in terms of detection capability.

This paper is structured as follows. Section II describes the problem addressed and the simulation set-up. Section III presents the analytical framework for the evaluation of accurate bounds for the probability of missed target detection. In Section IV we show simulation results, which confirm the validity of the proposed bounds. Finally, Section V concludes the paper. 


\section{SCEnARIO OF INTEREST}

\section{A. Problem Statement}

The surveillance of a given area is part of many military and civilian applications. WSNs can represent, for these applications, an efficient and flexible system to detect an incoming target in an area of interest. When unattended wireless sensors are used to monitor the given area, a naive solution consists in placing sensors all around the area. However, this solution is often practically unfeasible as, in most of the cases, either a limited number of deployable sensors is available or the area is too large for achieving complete coverage. Sensors have then to be placed efficiently to maximize the probability of detecting an intrusion.

In WSNs, battery-powered nodes are cyclically switched on and off, according to given duty cycles, typically at both sensing and communication levels. These power-saving operations raise a trade-off between system efficiency and energy consumption. In fact, by tuning the duty cycles, it is possible to extend the node lifetime, but, on the opposite, both the detection capability and the reactivity of a WSN reduce. In fact, the cyclical deactivation of sensing and communication interfaces tends to increase, respectively, the probability of missed target detection and the transmission latency of an "alert" message to a gateway node.

In the rest of this paper, we concentrate on the effect of sensing duty cycles on the probability of target detection. Assuming that a network operator deploys the available nodes in known positions (e.g., in the proximity of crossing areas, building perimeter, etc.), the problem is to assess the performance of the surveillance system (i.e., the probability of missed target detection) as a function of the nodes configuration (i.e., the sensing duty cycle), their number, and their relative positions. In this context, we derive upper and lower bounds for the probability of missing an incoming target. These bounds allow a network designer to evaluate the effectiveness of a specific node deployment for monitoring a critical area of interest.

\section{B. WSN Model}

The wireless sensor nodes considered in this paper are equipped with a seismic sensor, whose sensing range is $r_{\mathrm{s}}$ (dimension: [m]). To reduce the energy consumption of the system, the sensing part can be periodically switched off, according to a normalized duty cycle $\beta_{\text {sens }} \in[0,1]$ over a period $t_{\text {sens }}$ (dimension: [s]). More precisely, nodes sense the surrounding environment for an interval of length $\beta_{\text {sens }} t_{\text {sens }}$ and sleep for an interval of duration $\left(1-\beta_{\text {sens }}\right) t_{\text {sens }}$. This sensing/sleeping pattern repeats cyclically. We assume that all the sensors have the same $r_{\mathrm{s}}, \beta_{\mathrm{sens}}$, and $t_{\mathrm{sens}}$.

To make the derivation of the probability of target detection $P_{\mathrm{d}}$ (or, equivalently, of the probability of missed target detection $P_{\mathrm{md}}$ ) feasible, we assume the monitored area to be a square with sides of length $d_{\mathrm{s}}$ (dimension: [m]). In this area, $N$ sensors are placed in known positions, under the constraint that their sensing ranges do not overlap.

We assume that the potential targets cross the monitored area following linear trajectories. An illustrated example is

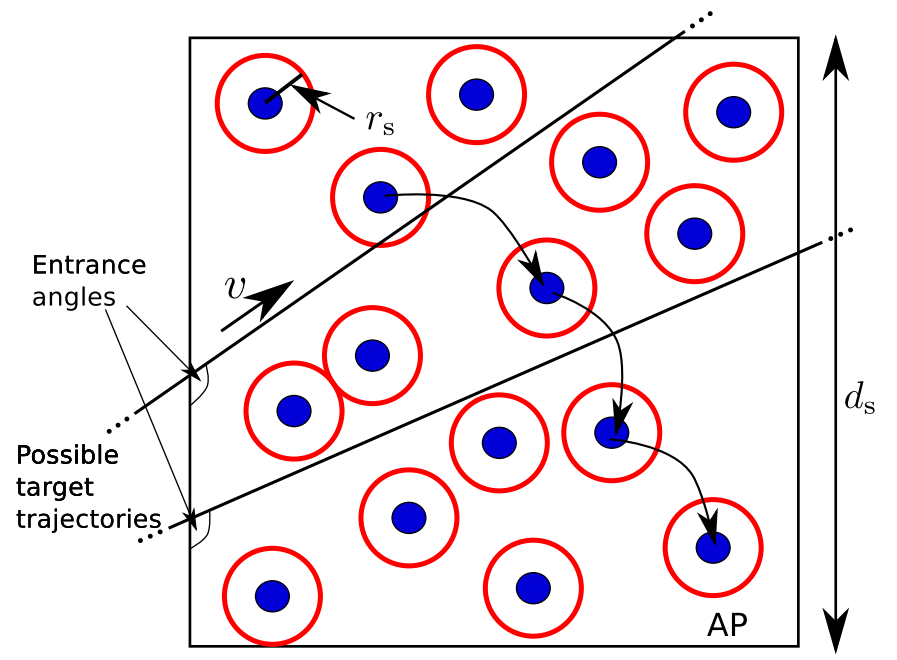

Figure 1. Illustrative example of network/node parameters.

shown in Figure 1. Each trajectory is characterized by (i) an entrance angle uniformly distributed in $[0, \pi]$ with respect to a reference axis given by the entrance side; and (ii) a constant target speed $v$ (dimension: $[\mathrm{m} / \mathrm{s}]$ ). Since there is no information about the entrance point, we also assume that the target entrance point into the monitored area is uniformly distributed over the perimeter of the monitored surface.

The main model parameters are set as follows: $d_{\mathrm{s}}=1000 \mathrm{~m}$, $v=15 \mathrm{~m} / \mathrm{s}, r_{\mathrm{s}}=50 \mathrm{~m}, t_{\text {sens }}=15 \mathrm{~s}$, and $N=5,10$ nodes.

\section{Probability of Target Missed Detection}

\section{A. Stochastic Node Deployment}

We now recall the basics of the model introduced in [1], relative to the assumption of an average node spatial distribution, i.e., a stochastic node deployment.

Assume that all the nodes have the same sensing area of perimeter $l_{i}=2 \pi r_{\mathrm{s}}(i=1, \ldots, N)$ and that the nodes are randomly deployed over a square area with perimeter $l_{0}=4 d_{\mathrm{s}}$ (dimension: [m]). The probability of detecting a target is the probability that there is a sensor on the target trajectory (event denoted as $\mathcal{E}_{\mathrm{SoT}}$ ) and that the sensor is active when the target is crossing the sensed area (event denoted as $\mathcal{E}_{\text {det }}$ ). Therefore, the probability that a single sensor detects a target, named $P_{\mathrm{d}}^{(1)}$, is

$$
P_{\mathrm{d}}^{(1)}=P\left\{\mathcal{E}_{\mathrm{SoT}}, \mathcal{E}_{\mathrm{det}}\right\}=P\left\{\mathcal{E}_{\mathrm{det}} \mid \mathcal{E}_{\mathrm{SoT}}\right\} P\left\{\mathcal{E}_{\mathrm{SoT}}\right\} .
$$

According to the results in [5], $P\left\{\mathcal{E}_{\mathrm{SoT}}\right\}$ can be expressed as $2 \pi r_{\mathrm{s}} / 4 d_{\mathrm{s}}$. In order to evaluate $P\left\{\mathcal{E}_{\mathrm{det}} \mid \mathcal{E}_{\mathrm{SoT}}\right\}$, we consider the scheme for the sleeping duty cycle shown in Fig. 2 (a). Since the target arrives with a finite speed $v$, the crossing time is $T_{\text {cross }}=L / v$, where $L$ is a random variable which expresses the length of the intersection between the target trajectory and the area sensed by a sensor, as shown in Fig. 2 (b). Since there is no information about the arrival instant of the target, we assume it to be uniformly distributed over a period of duration $t_{\text {sens }}$. 


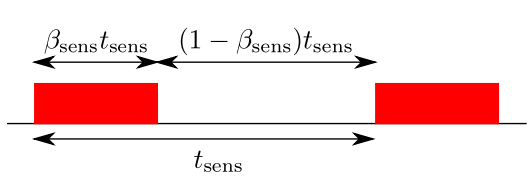

(a)



(b)

Figure 2. (a) Logical scheme of the sensing duty cycle and (b) model for the sensing range of a node.

We now focus on a single period of duration $t_{\text {sens }}$. When the sensor is on, i.e., during the subinterval of duration $\beta_{\text {sens }} t_{\text {sens }}$, any incoming target will be detected. In the case that the sensor is off, i.e., during the subinterval of duration $\left(1-\beta_{\text {sens }}\right) t_{\text {sens }}$, the following analysis can be carried out. Let $\mathcal{E}_{\text {target }}$ be the event $\{$ The sensor is on at the instant at which the target enters the sensed area\}. Applying the total probability theorem [6], $P\left\{\mathcal{E}_{\mathrm{det}} \mid \mathcal{E}_{\mathrm{SoT}}\right\}$ can then be expressed as

$P\left\{\mathcal{E}_{\text {det }} \mid \mathcal{E}_{\text {SoT }}\right\}=P\left\{\mathcal{E}_{\text {det }} \mid \mathcal{E}_{\text {target }}, \mathcal{E}_{\text {SoT }}\right\} P\left\{\mathcal{E}_{\text {target }} \mid \mathcal{E}_{\text {SoT }}\right\}$

$$
+P\left\{\mathcal{E}_{\text {det }} \mid \overline{\mathcal{E}}_{\text {target }}, \mathcal{E}_{\text {SoT }}\right\} P\left\{\overline{\mathcal{E}}_{\text {target }} \mid \mathcal{E}_{\text {SoT }}\right\}(2)
$$

where it is immediate to conclude that $P\left\{\mathcal{E}_{\text {det }} \mid \mathcal{E}_{\text {target }}, \mathcal{E}_{\text {SoT }}\right\}=1, P\left\{\mathcal{E}_{\text {target }} \mid \mathcal{E}_{\text {SoT }}\right\}=\beta_{\text {sens }}$, and $P\left\{\overline{\mathcal{E}}_{\text {target }} \mid \mathcal{E}_{\mathrm{SoT}}\right\}=1-\beta_{\text {sens }}$. Therefore, equation (2) can be rewritten as:

$$
P\left\{\mathcal{E}_{\text {det }} \mid \mathcal{E}_{\text {SoT }}\right\}=\beta_{\text {sens }}+\left(1-\beta_{\text {sens }}\right) \mathrm{P}\left\{\mathcal{E}_{\text {det }} \mid \overline{\mathcal{E}}_{\text {target }}, \mathcal{E}_{\text {SoT }}\right\} .
$$

The last remaining term at the right-hand side of (2) can be expressed as [1]:

$$
P\left\{\mathcal{E}_{\text {det }} \mid \overline{\mathcal{E}}_{\text {target }}, \mathcal{E}_{\text {SoT }}\right\}=\iint_{\mathcal{D}} f_{T_{\mathrm{a}}, T_{\text {cross }}}(t, \tau) \mathrm{d} t \mathrm{~d} \tau
$$

where the domain $\mathcal{D}$ is described in [1, Figure 1] and the joint probability density function (pdf) $f_{T_{\mathrm{a}}, T_{\text {cross }}}(t, \tau)$ can be expressed as [1]:

$$
\begin{aligned}
& f_{T_{\mathrm{a}}, T_{\text {cross }}}(t, \tau)= \\
& \left\{\begin{array}{cc}
\frac{v}{\pi c \sqrt{r_{\mathrm{s}}^{2}-\left(\frac{v \tau}{2}\right)^{2}}} & \text { if } 0<\tau<2 r_{\mathrm{s}} / v, \\
0 & 0<t<c
\end{array}\right. \\
& \hline
\end{aligned}
$$

where $c \triangleq\left(1-\beta_{\text {sens }}\right) t_{\text {sens. }}$. At this point, given that the arrival time $T_{\mathrm{a}}$ of the target is Unif $[0, c]$, it can show that:

$$
\begin{gathered}
P\left\{\mathcal{E}_{\text {det }} \mid \overline{\mathcal{E}}_{\text {target }}, \mathcal{E}_{\mathrm{SoT}}\right\}= \\
\begin{cases}\frac{4 r_{\mathrm{s}}}{\pi c v} & \text { if } 2 r_{\mathrm{s}} / v<c \\
\frac{4 r_{\mathrm{s}}-2 \sqrt{4 r_{\mathrm{s}}^{2}-c^{2} v^{2}}}{\pi c v}+1-\frac{2 \operatorname{asin}\left(\frac{c v}{2 r_{\mathrm{s}}}\right)}{\pi} & \text { otherwise. }\end{cases}
\end{gathered}
$$

Taking into account the independence between the $N$ randomly deployed sensors, the probability of (network) missed target detection becomes

$$
\begin{aligned}
& P_{\mathrm{md}}=\left(1-P_{\mathrm{d}}^{(1)}\right)^{N} \\
= & \left(1-\left[\beta_{\text {sens }}+\left(1-\beta_{\text {sens }}\right) P\left\{\mathcal{E}_{\text {det }} \mid \overline{\mathcal{E}}_{\text {target }}, \mathcal{E}_{\mathrm{SoT}}\right\}\right] \frac{2 \pi r_{\mathrm{s}}}{4 d_{\mathrm{s}}}\right)^{N}(6)
\end{aligned}
$$

where $P\left\{\mathcal{E}_{\text {det }} \mid \overline{\mathcal{E}}_{\text {target }}, \mathcal{E}_{\text {SoT }}\right\}$ has the expression (5). Expression (6) is valid when there is no information about node positions. However, its accuracy can be significantly improved if the positions of the nodes are known, i.e., with a deterministic deployment. This scenario is considered in the following subsection.

\section{B. Deterministic Node Deployment}

In the case of deterministic deployment, it is possible, yet cumbersome, to obtain an exact expression of the average probability of detection. However, we now show how to derive accurate upper and lower bounds.

Given $N$ sensors placed in known positions with $\beta_{\text {sens }}=1$, the probability of target detection can be expressed as [5]:

$$
P_{\mathrm{d}}=1-P_{\mathrm{md}}=P\left(\ell \cap \bigcup_{i=1}^{N} \mathcal{A}_{i}\right)
$$

where $\ell$ is a generic line crossing the monitored surface, and $\mathcal{A}_{i}$ are the area sensed by node $i$. The expression at the righthand side of (7) can be rewritten, using the Feller's inclusionsexclusion principle [7], as the sum of joint probabilities of a line intersecting specific set arrangements:

$$
\begin{aligned}
P_{\mathrm{d}}=\quad & \sum_{i=1}^{N} P\left(\ell \cap \mathcal{A}_{i} \neq 0\right)-\sum_{i, j: i<j}^{N} P\left(\ell \cap \mathcal{A}_{i} \cap \mathcal{A}_{j} \neq 0\right) \\
& +\cdots+(-1)^{N} P\left(\ell \cap \mathcal{A}_{1} \cap \mathcal{A}_{2} \cdots \mathcal{A}_{N}\right)
\end{aligned}
$$

The expression at the right-hand side of (8) is hard to compute, since it requires information on the probability that a generic line crosses all sensors' subsets. For instance, considering the first term at the right-hand side of (8), the subset is formed by one sensor, whereas considering the last term, the subset is formed by the $N$ nodes in the network. Therefore, it is preferable to compute the following upper and lower bounds, simply obtained by considering only a few terms among those in expression (8):

$$
\begin{aligned}
\sum_{i=1}^{N} \underbrace{P\left(\ell \cap \mathcal{A}_{i} \neq 0\right)}_{\triangleq m_{1}(i) / l_{0}}-\sum_{i, j: i<j}^{N} \underbrace{P\left(\ell \cap \mathcal{A}_{i} \cap \mathcal{A}_{j} \neq 0\right)}_{\triangleq m_{2}(i, j) / l_{0}}< \\
<P_{\mathrm{d}}<\sum_{i=1}^{N} \underbrace{P\left(\ell \cap \mathcal{A}_{i} \neq 0\right)}_{=m_{1}(i) / l_{0}}
\end{aligned}
$$

where $l_{0}=4 d_{\mathrm{s}}$ is the perimeter of the monitored area.

As previously introduced, the bounds in (9) refer to the case that sensors are always switched on, i.e., with $\beta_{\text {sens }}=1$. We now extend the bounds (9) in order to take into account the impact of the duty cycle. Since $m_{1}(i)=2 \pi r_{\mathrm{s}}, \forall i$ is the probability that a generic line crosses a sensed area and according to the considerations in Subsection III-A, the duty cycle can be taken into account by simply scaling this term by the probability $P\left\{\mathcal{E}_{\mathrm{det}} \mid \mathcal{E}_{\mathrm{SoT}}\right\}$, and thus deriving the following upper bound

$$
\sum_{i=1}^{N} \frac{m_{1}(i)}{l_{0}} \cdot P\left\{\mathcal{E}_{\mathrm{det}} \mid \mathcal{E}_{\mathrm{SoT}}\right\}=N \frac{2 \pi r_{\mathrm{s}}}{4 d_{\mathrm{s}}} \cdot P\left\{\mathcal{E}_{\mathrm{det}} \mid \mathcal{E}_{\mathrm{SoT}}\right\}
$$


Therefore, the computation of the term $\left\{m_{1}(i)\right\}$ does not require information about node positions, and the scaling factor $P\left\{\mathcal{E}_{\mathrm{det}} \mid \mathcal{E}_{\mathrm{SoT}}\right\}$ can be evaluated according to (3).

The term $m_{2}(i, j)$, instead, represents the probability that the target trajectory crosses both the areas sensed by nodes $i$ and $j$. In this case, both nodes $i$ and $j$ must be active when the target is crossing its sensed area, in order to detect the target. We point out that the computation of $m_{2}$, unlike that of $m_{1}$, requires information about the (relative) node positions.

The second term of the lower bound in (8) can then be extended as

$$
\sum_{i, j: i<j} \frac{m_{2}(i, j)}{4 d_{\mathrm{s}}} \cdot P_{2}\left\{\mathcal{E}_{\mathrm{det}} \mid \mathcal{E}_{\mathrm{SoT}_{i, j}}\right\}
$$

where $P_{2}\left\{\mathcal{E}_{\mathrm{det}} \mid \mathcal{E}_{\mathrm{SoT}_{i, j}}\right\}$ is the probability that the target is detected during the active phase of both two sensors, given that the target trajectory crosses both sensed areas. Since the target trajectory is random and there is no synchronization between the two sensors, the detection carried out by sensor $i$ is independent from the one carried out by sensor $j$, and $P_{2}\left\{\mathcal{E}_{\mathrm{det}} \mid \mathcal{E}_{\mathrm{SoT}_{i, j}}\right\}$ can be expressed as

$$
P_{2}\left\{\mathcal{E}_{\mathrm{det}} \mid \mathcal{E}_{\mathrm{SoT}_{i, j}}\right\}=P_{2}^{(i)}\left\{\mathcal{E}_{\mathrm{det}} \mid \mathcal{E}_{\mathrm{SoT}_{i}}\right\} \cdot P_{2}^{(j)}\left\{\mathcal{E}_{\mathrm{det}} \mid \mathcal{E}_{\mathrm{SoT}_{j}}\right\}
$$

where $P_{2}^{(i)}\left\{\mathcal{E}_{\mathrm{det}} \mid \mathcal{E}_{\mathrm{SoT}_{i}}\right\}$ is the probability that node $i$ is active, given that the target crosses its sensed area, and $P_{2}^{(j)}\left\{\mathcal{E}_{\mathrm{det}} \mid \mathcal{E}_{\mathrm{SoT}_{j}}\right\}$ is the probability that node $j$ is active, given that the target crosses its sensed area.

The allowed trajectories for the computation of $m_{2}$ (i.e., the trajectories which belong to both sensed areas) are a subset of all admissible ones for a single sensor shown in Figure 3 (a). In fact, in the case of $m_{1}$, the entrance point of the target in the area sensed by a sensor can be the whole perimeter of the sensed area, so that the angle $\Phi$, shown in Figure 2 (b), can range in the interval $[0,2 \pi]$. Conversely, in the $m_{2}$ case, one must consider, for node $i$, only the entrance points which allow the target to cross both sensed areas. Referring to the logical scheme shown in Figure 3 (a), the range of allowed entrance points for the first sensed area can be approximated as the arc, highlighted in Figure 3 (a), which insists on the angle $\left(2 \pi-2 \theta_{\lim _{1}}\right)$, where $\theta_{\lim _{1}}$ is a function of $d_{i, j}$ (the distance between sensor $i$ and sensor $j$ ) and $r_{\mathrm{s}}$.

In order to simplify the derivation of $P_{2}^{(i)}\left\{\mathcal{E}_{\mathrm{det}} \mid \mathcal{E}_{\mathrm{SoT}_{i}}\right\}$, we must find the distribution of the length $L_{i}^{(i, j)}$ of the intersection between the target trajectory and the area sensed by node $i$. Moreover, one also needs to compute, for node $i$, the joint pdf $f_{T_{\mathrm{a}}, T_{\text {cross }}}^{(i)}(t, \tau)$, which replaces that presented in expression (4). Similar considerations can be carried out for the derivation of $P_{2}^{(j)}\left\{\mathcal{E}_{\mathrm{det}} \mid \mathcal{E}_{\mathrm{SoT}_{j}}\right\}$ which, as shown in Figure 3 (b), depends on the entrance point of the node in sensor $j$. Assuming that the target has already crossed the area sensed by node $i$, the range of possible entrance points lies on the arc, highlighted in Figure 3 (b), which insists on the angle $\left(2 \pi-2 \theta_{\lim _{2}}\right)$. As for node $i$, we simplify the derivation of the distribution of the length $L_{j}^{(i, j)}$ of the intersection between the target trajectory and the area sensed by $j$. In particular,

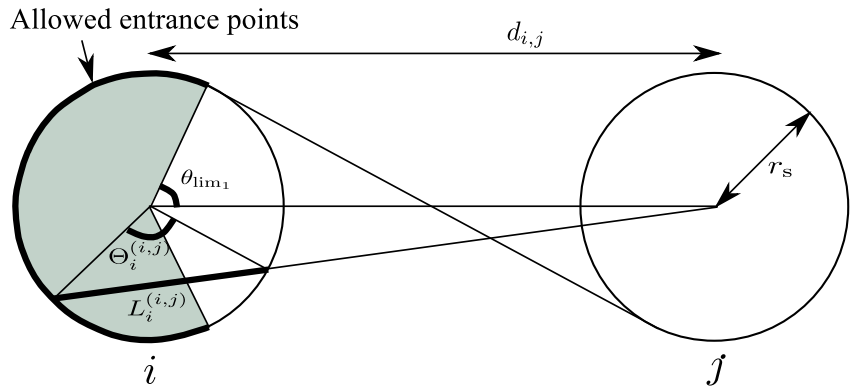

(a)

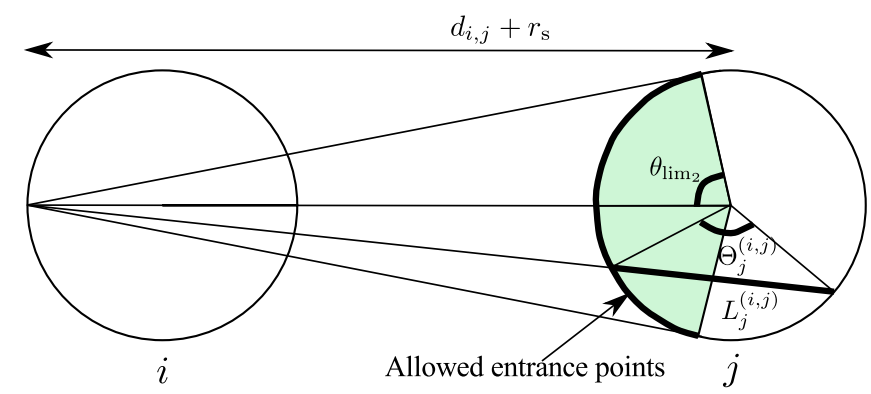

(b)

Figure 3. Allowed entrance points: (a) in sensor $i$, which ensure that the sensor $j$ can be crossed by the target, and (b) in sensor $j$, given that the target has already crossed the sensor $i$.

referring to Figure 3 (b), we assume that all the targets enter in the area sensed by node $i$ in the point lying on the line connecting the nodes $i$ and $j$.

On the basis of geometrical considerations, omitted here for lack of space, the length of a chord $L$ can be expressed as a function of the angle $\Theta$ as follows: ${ }^{1}$

$$
\begin{aligned}
L=b_{1}-\left\{r_{\mathrm{s}}^{2}+d^{2}-2 r_{\mathrm{s}} d\left[-\left\{\left(1-\frac{d \sin \Theta}{b_{1}}\right)\right.\right.\right. \\
\left.\left.\left.\cdot\left(1-\frac{r_{\mathrm{s}} \sin \Theta}{b_{1}}\right)\right\}^{1 / 2}+\frac{r_{\mathrm{s}} d \sin ^{2} \Theta}{b_{1}^{2}}\right]\right\}^{1 / 2}(12)
\end{aligned}
$$

where $b_{1} \triangleq \sqrt{r_{\mathrm{s}}^{2}+d^{2}-2 r_{\mathrm{s}} d \cos \Theta}$, and

$$
d=\left\{\begin{array}{cc}
d_{i, j} & \text { if } L=L_{i}^{(i, j)} \\
d_{i, j}+r_{\mathrm{s}} & \text { if } L=L_{j}^{(i, j)}
\end{array}\right.
$$

Since expression (12) is complicated, it can be shown that it can be accurately approximated through a degree- 2 polynomial approximation. More precisely, assuming that

$$
L \simeq a_{1} \Theta^{2}+a_{2} \Theta+a_{3},
$$

where $a_{1}, a_{2}$, and $a_{3}$ are shown in Table I, it is possible to evaluate the joint pdf which replaces that of equation $(4)^{2}$. This

\footnotetext{
${ }^{1}$ According to Figure 3 , we should compute $L_{i}^{(i, j)}$ and $L_{j}^{(i, j)}$ by considering the angles $\Theta_{i}^{(i, j)}$ and $\Theta_{j}^{(i, j)}$, respectively. However, since the expression is the same in for both node $i$ and node $j$ and for ease of notation simplification, we refer to $L$ and $\Theta$, considering a generic pair of sensors.

${ }^{2}$ As for expression (12), for ease of notation simplification, we refer to $\theta_{\mathrm{lim}}$, instead of $\theta_{\lim _{1}}$ and $\theta_{\lim _{2}}$.
} 


\begin{tabular}{|c|c|c|}
\hline Coefficients & Analytical expressions & Numerical values \\
\hline$a_{1}$ & $-\frac{2 r_{\mathrm{s}}}{\left(\pi-\theta_{\mathrm{lim}}\right)^{2}}$ & -38.06 \\
\hline$a_{2}$ & $-2 a_{1} \pi$ & 239.17 \\
\hline$a_{3}$ & $a_{1} \pi^{2}+2 r_{\mathrm{s}}$ & -275.69 \\
\hline
\end{tabular}

Table I

COEFFICIENTS FOR THE POLYNOMIAL APPROXIMATION OF (14).

equation should depend on the distance between nodes $i$ and $j$. However, after some algebraic manipulations, the dependence on the distance between the pair of sensors disappears and it can be shown that:

$$
\begin{gathered}
f_{T_{\mathrm{a}}, T_{\text {cross }}}^{(i)}(t, \tau)=f_{T_{\mathrm{a}}, T_{\text {cross }}}^{(j)}(t, \tau) \\
= \begin{cases}\frac{v}{c \sqrt{16 r_{\mathrm{s}}^{2}-8 r_{\mathrm{s}} v \tau}} & \text { if } 0<\tau<\frac{2 r_{\mathrm{s}}}{v}, \\
0 & 0<t<c\end{cases} \\
\text { otherwise. }
\end{gathered}
$$

As for the random node deployment case in [1], the evaluation of $P_{2}^{(i)}\left\{\mathcal{E}_{\mathrm{det}} \mid \mathcal{E}_{\mathrm{SoT}_{i}}\right\}$ depends on the relation between $2 r_{\mathrm{s}} / v$ and $\left(1-\beta_{\text {sens }}\right) t_{\text {sens }}$, but not on node $i$. In particular, it can be shown that

$$
\begin{gathered}
P\left\{\mathcal{E}_{\text {det }} \mid \overline{\mathcal{E}}_{\text {target }}, \mathcal{E}_{\mathrm{SoT}_{i}}\right\}=P\left\{\mathcal{E}_{\text {det }} \mid \overline{\mathcal{E}}_{\text {target }}, \mathcal{E}_{\mathrm{SoT}_{j}}\right\} \\
=\left\{\begin{array}{lr}
\frac{4 r_{\mathrm{s}}}{3 c v} & \text { if } 2 r_{\mathrm{s}} / v<c \\
\frac{4 r_{\mathrm{s}}}{3}-\frac{\left(c v+4 r_{\mathrm{s}}\right) \sqrt{16 r_{\mathrm{s}}^{2}-8 c r_{\mathrm{s}} v}}{12 r_{\mathrm{s}}} & \frac{\sqrt{16 r_{\mathrm{s}}^{2}-8 r_{\mathrm{s}} c v}}{4 r_{\mathrm{s}}} \\
& \begin{array}{c}
\text { otherwise. }
\end{array}
\end{array}\right.
\end{gathered}
$$

Since, at this point, $P_{2}^{(i)}\left\{\mathcal{E}_{\mathrm{det}} \mid \mathcal{E}_{\mathrm{SoT}_{i}}\right\}=P_{2}^{(j)}\left\{\mathcal{E}_{\mathrm{det}} \mid \mathcal{E}_{\mathrm{SoT}_{j}}\right\}$, we can rewrite expression (11) as:

$$
P_{2}\left\{\mathcal{E}_{\mathrm{det}} \mid \mathcal{E}_{\mathrm{SoT}_{i, j}}\right\}=\left(P_{2}^{(i)}\left\{\mathcal{E}_{\mathrm{det}} \mid \mathcal{E}_{\mathrm{SoT}_{i}}\right\}\right)^{2} .
$$

At this point, for the computation of expression (10), we just need to compute $m_{2}(i, j)$. This term can be evaluated, as shown in [5], as a function of the distance between each couple of sensor nodes [8]. In particular, considering equal sensing ranges, $m_{2}(i, j)$ can be expressed as

$$
m_{2}(i, j)=\left\{\begin{array}{cc}
2 \pi r_{\mathrm{s}}+2 \pi r_{\mathrm{s}}-L_{\mathrm{out}}\left(d_{i, j}\right), & \mathcal{A}_{i} \cap \mathcal{A}_{j} \neq 0 \\
L_{\text {in }}\left(d_{i, j}\right)-L_{\text {out }}\left(d_{i, j}\right) & \mathcal{A}_{i} \cap \mathcal{A}_{j}=0
\end{array}\right.
$$

where (after some geometrical considerations)

$$
\begin{aligned}
& L_{\text {out }}\left(d_{i, j}\right)=2 \pi r_{\mathrm{s}}+2 d_{i, j} \\
& L_{\text {in }}\left(d_{i, j}\right)=2 r_{\mathrm{s}}\left[2 \pi-2 \arccos \left(\frac{2 r_{\mathrm{s}}}{d_{i, j}}\right)\right]+4 \sqrt{\frac{d_{i, j}^{2}}{4}-r_{\mathrm{s}}^{2}} .
\end{aligned}
$$

At this point, the bounds in (9) can be evaluated, thus

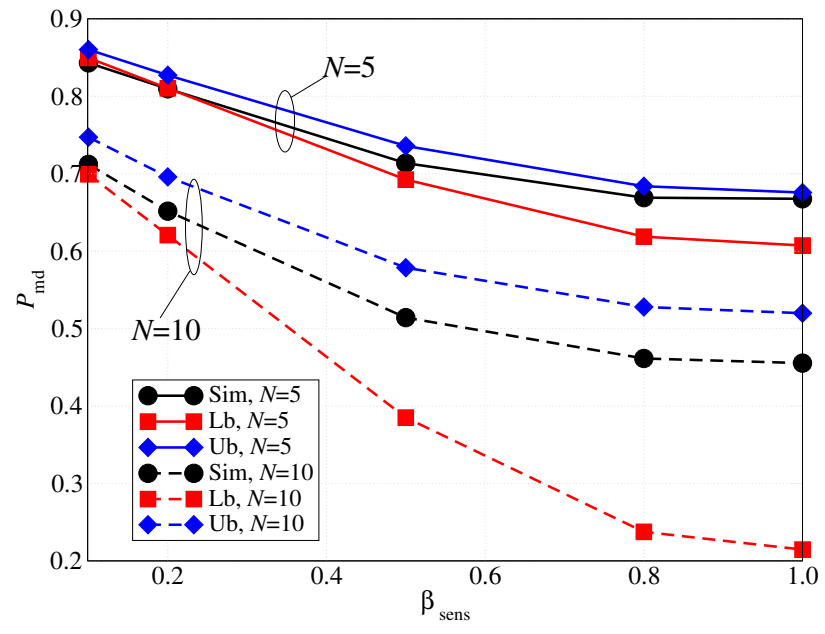

Figure 4. $\quad P_{\mathrm{md}}$ as a function of $\beta_{\text {sens }}$, considering deterministic deployment with (i) $N=5$ nodes and (ii) $N=10$ nodes deployed over a square surface.

obtaining:

$$
\begin{aligned}
& 1-\sum_{i=1}^{N} \frac{m_{1}(i)}{4 d_{\mathrm{s}}} P\left\{\mathcal{E}_{\mathrm{det}} \mid \mathcal{E}_{\mathrm{SoT}}\right\}<P_{\mathrm{md}}<1-\sum_{i=1}^{N} \frac{m_{1}(i)}{4 d_{\mathrm{s}}} \\
& \cdot P\left\{\mathcal{E}_{\mathrm{det}} \mid \mathcal{E}_{\mathrm{SoT}}\right\}+\sum_{i, j: i<j}^{N} \frac{m_{2}(i, j)}{4 d_{\mathrm{s}}} P_{2}\left\{\mathcal{E}_{\mathrm{det}} \mid \mathcal{E}_{\mathrm{SoT}_{i, j}}\right\} .
\end{aligned}
$$

\section{Performance Evaluation}

In this section, we analyze the accuracy of the proposed analytical framework through simulations. We consider node deployments over a squared area and use parameters presented in Subsection II-B. To evaluate the probability of missed target detection, we average the results over 1000 different topologies and, for each topology, we consider 1000 different target trajectories.

In Figure $4, P_{\mathrm{md}}$ is shown as a function of $\beta_{\mathrm{sens}}$. Considering the curves relatives to $N=5$, the upper and lower bounds are quite close to each other. In fact, since there are only a few nodes in the network, it is likely that a target crosses only one or, at most, two sensed areas. In this case, the performance is well approximated by the $m_{1}$ and $m_{2}$ terms computed as described in Subsection III-B. When $\beta_{\text {sens }}$ is small, the simulated values of $P_{\mathrm{md}}$ are slightly below the lower bound. In fact, if it happens that three sensors lie on the same target trajectory, the calculation of the term $m_{1}$ is not accurate enough to well approximate the true value of $P_{\mathrm{md}}$. In order to improve the accuracy of the lower bound, the computation of the contribution of the higher-order terms in expression (8) would be required. However, from a network designer perspective, it is sufficient that the upper bound is accurate. More precisely, as in [1], in the current case (with deterministic deployment) one could minimize the upper bound for $P_{\mathrm{md}}$, thus guaranteeing that, for each considered topology, the real $P_{\mathrm{md}}$ performance will be better than that predicted by the bound.

Considering the case with $N=10$ nodes, instead, the simulated performance lies inside the bounds for all values of $\beta_{\text {sens. }}$. In this case, however, the lower bound is quite 


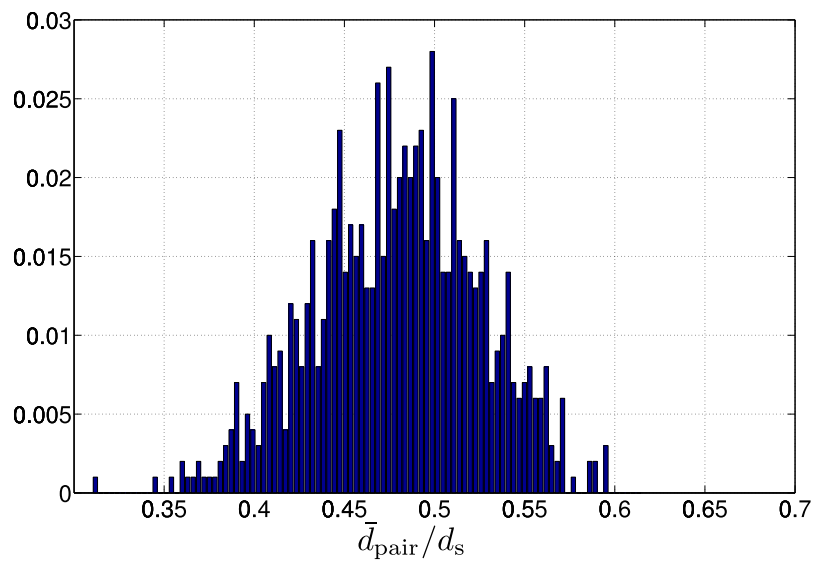

(a)

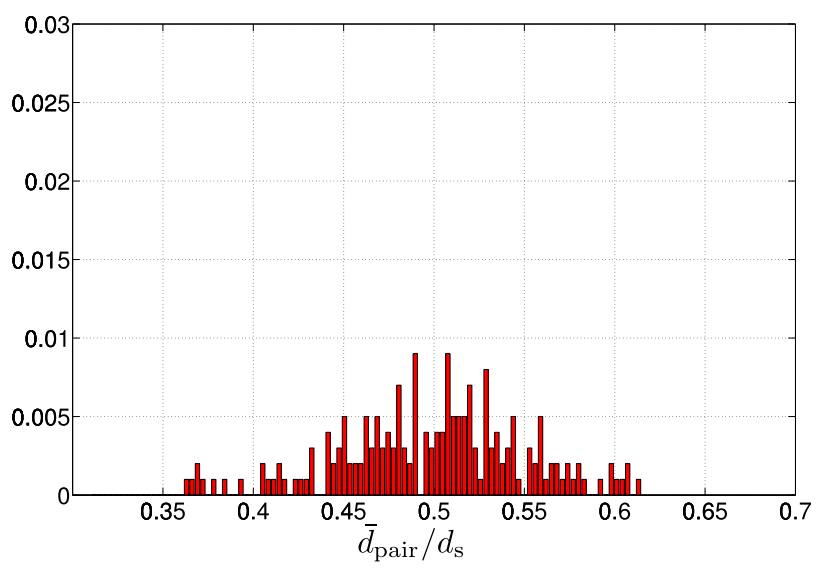

(b)

Figure 5. Normalized number of times that the simulated $P_{\mathrm{md}}$ lies (a) inside or (b) outside the bounds for all values of $\beta_{\text {sens }}$. The number of nodes is $N=10$.

coarse. In fact, it is more likely that there are three or more sensors on the same trajectory, so that the computation of only the $m_{1}(i)$ terms is not sufficient to correctly estimate $P_{\text {md }}$. However, the upper bound remains quite close to the simulation performance. In fact, the presence of the $m_{2}(i, j)$ terms allows to better approximate $P_{\mathrm{md}}$.

Due to the dependence of $P_{\mathrm{md}}$ on the node positions, it is of interest to analyze the impact of the nodes spatial distribution. To do this, we consider the average distance, denoted as $\bar{d}_{\text {pair }}$, between all possible pairs of nodes. For each generated topology and associated value of $\bar{d}_{\text {pair }}$, we compute the upper and lower bounds and check if the simulation-based (averaged out over 1000 runs) value of $P_{\text {md }}$ lies between the bounds. We consider 1000 topologies and count, for each value of $\bar{d}_{\text {pair }} / d_{\mathrm{s}}$ (quantized in intervals of length 0.03 ), the number of cases where the obtained performance lies/does not lie between the bounds. The results are shown in Figure 5 (a) and (b), in the cases where the simulation results lie/do not lie within the bounds, respectively. As one can see, the simulated $P_{\text {md }}$ lies outside the bounds only in a few cases, confirming that the validity of the proposed analytical framework.

On the basis of other results (not presented here for lack of space), the following conclusions can be carried out.

- For small values of $N$, the number of topologies, whose simulation-based value of $P_{\mathrm{md}}$, is outside the bounds is larger. In particular, by considering the node spatial density $\rho_{\mathrm{s}} \triangleq N / d_{\mathrm{s}}^{2}$ (dimension: [nodes $\left./ \mathrm{m}^{2}\right]$ ), it can be concluded that, for $\rho_{\mathrm{s}}>5 / 1000^{2}=5 \cdot 10^{-6}$ nodes $/ \mathrm{m}^{2}$, the framework allows to accurately predict the probability of missed target detection in the considered scenarios with $r_{\mathrm{s}}=50 \mathrm{~m}$.

- For small values of $\beta_{\text {sens }}$, it is more likely that $P_{\mathrm{md}}$ lies outside the bounds.

- Considering only the topologies whose performance lies below the upper bound, the approximation works better, allowing a good performance prediction.

\section{CONCLUDING REMARKS}

This paper has addressed the problem of evaluating the probability of detecting a target crossing a given area where $N$ sensing nodes are deployed. The wireless devices use a sensing duty cycle to save energy. Our focus has been on the performance analysis with deterministic nodes placement. In this context, we have derived accurate upper and lower bounds on the probability of missed target detection. The simulation results confirm that the derived bounds become very accurate when the node spatial density is above a minimum value (on the order of 5 nodes per $\mathrm{Km}^{2}$ in the case with $r_{\mathrm{s}}=50 \mathrm{~m}$ ).

\section{ACKNOWLEDGEMENTS}

This work has been partly funded by the French ANR SenLab project and the European ITEA GEODES project.

\section{REFERENCES}

[1] P. Medagliani, J. Leguay, V. Gay, M. Lopez-Ramos, and G. Ferrari, "Engineering energy-efficient target detection applications in wireless sensor networks," in Proc. 8th IEEE Int. Conf. on Pervasive Computing and Communications (PerCom 2010), Mannheim, Germany, April 2010, 9 pages.

[2] T. He, S. Krishnamurthy, L. Luo, T. Yan, L. Gu, R. Stoleru, G. Zhou, Q. Cao, P. Vicaire, J. A. Stankovic, T. F. Abdelzaher, J. Hui, and B. Krogh, "Vigilnet: An integrated sensor network system for energyefficient surveillance," ACM Trans. Sen. Netw., vol. 2, no. 1, pp. 1-38, February 2006.

[3] J. Jeong, Y. Gu, T. He, and D. Du, "VISA: Virtual Scanning Algorithm for Dynamic Protection of Road Networks," in Proc. of 28th IEEE Conference on Computer Communications (INFOCOM 09), Rio de Janeiro, Brazil, April 2009, 9 pages.

[4] H. Zhang and J. Hou, "Is deterministic deployment worse than random deployment for wireless sensor networks?" in Proc. of 25th IEEE Conference on Computer Communications (INFOCOM 06), Barcelona, Spain, April 2006, 13 pages.

[5] L. Lazos, R. Poovendran, and J. A. Ritcey, "Analytic evaluation of target detection in heterogeneous wireless sensor networks," ACM Trans. Sensor Networks, vol. 5, no. 2, pp. 1-38, March 2009.

[6] A. Papoulis, Probability, Random Variables and Stochastic Processes. New York, NJ, USA: McGraw-Hill, 1991.

[7] W. Feller, "An introduction to probability theory and its applications," New York, NJ, USA, 1971.

[8] J. Sylvester, "On a funicular solution of Buffon's "problem of the needle" in its most general form," Acta Mathematica, vol. 14, no. 1, pp. 185-205, 1890 . 